University of Wollongong

Research Online

Faculty of Engineering and Information

Faculty of Engineering and Information

Sciences - Papers: Part B

Sciences

2020

\title{
Multi-period data driven control strategy for real-time management of energy storages in virtual power plants integrated with power grid
}

\author{
Chixin Xiao \\ University of Wollongong, cx472@uowmail.edu.au \\ Danny Sutanto \\ University of Wollongong, soetanto@uow.edu.au \\ Kashem M. Muttaqi \\ University of Wollongong, kashem@uow.edu.au \\ Minjie Zhang \\ University of Wollongong, minjie@uow.edu.au
}

Follow this and additional works at: https://ro.uow.edu.au/eispapers1

Part of the Engineering Commons, and the Science and Technology Studies Commons 


\title{
Multi-period data driven control strategy for real-time management of energy storages in virtual power plants integrated with power grid
}

\author{
Abstract \\ 2019 Elsevier Ltd This paper investigates a novel real-time stochastic multi-period management strategy \\ of a virtual power plant (VPP) using a three-layer language protocol based on computer program compiler \\ principle, which takes advantage of the availability of the battery storage in a VPP to maximize the \\ revenue of the VPP over the entire trading horizon considering the predicted prices in each slice of that \\ horizon. When the conventional scenario tree method is used to solve the computational complexity of \\ the multi-period stochastic optimization problem, it may cause the problem to become intractable when \\ the problem-scale increases. This paper proposes a deterministic lookahead approach that makes use of \\ a novel formal language that implements a special formal grammar to manage the real-time control on \\ the battery storages of the VPP. The control of charging/discharging of the battery storages, which is \\ driven by the real-time spot price and the rolling price prediction, is formalized by using the proposed \\ recursive grammar and the corresponding non-deterministic finite automaton (NFA). For validation, the \\ proposed approach is applied to a simple three-bus and an adapted IEEE 14-bus test system. The \\ simulation results show that the proposed method can obtain optimal revenue by managing each battery \\ in the VPP to operate as a local generator, a local load, an energy buyer, an energy seller, or by being in an \\ idle state when the battery is full or empty. \\ Disciplines \\ Engineering | Science and Technology Studies

\section{Publication Details} \\ C. Xiao, D. Soetanto, K. Muttaqi \& M. Zhang, "Multi-period data driven control strategy for real-time \\ management of energy storages in virtual power plants integrated with power grid," International Journal \\ of Electrical Power and Energy Systems, vol. 118, 2020.
}




\title{
Multi-Period Data Driven Control Strategy for Real-Time Management of Energy Storages in Virtual Power Plants Integrated with Power Grid
}

\author{
Chixin Xiao, Member IEEE, Danny Sutanto, Senior Member, IEEE, Kashem M. Muttaqi, Senior \\ Member, Minjie Zhang, Senior Member IEEE
}

\begin{abstract}
This paper investigates a novel real-time stochastic multi-period management strategy of a virtual power plant (VPP) using a three-layer language protocol based on computer program compiler principle, which takes advantage of the availability of the battery storage in a VPP to maximize the revenue of the VPP over the entire trading horizon considering the predicted prices in each slice of that horizon. When the conventional scenario tree method is used to solve the computational complexity of the multi-period stochastic optimization problem, it may cause the problem to become intractable when the problem-scale increases. This paper proposes a deterministic lookahead approach that makes use of a novel formal language that implements a special formal grammar to manage the real-time control on the battery storages of the VPP. The control of charging/discharging of the battery storages, which is driven by the real-time spot price and the rolling price prediction, is formalized by using the proposed recursive grammar and the corresponding non-deterministic finite automaton (NFA). For validation, the proposed approach is applied to a simple three-bus and an adapted IEEE 14-bus test system. The simulation results show that the proposed method can obtain optimal revenue by managing each battery in the VPP to operate as a local generator, a local load, an energy buyer, an energy seller, or by being in an idle state when the battery is full or empty.
\end{abstract}

Index Terms - time-staged optimization, energy storage, nondeterministic finite automaton, formal grammar, price-driven, renewable energy sources, dynamic economic dispatch.

\section{NOMENCLATURE}

$R^{t}: \quad$ The amount of energy in the battery at time $t$ (MW)

$R_{j}^{t}$ : $\quad$ The amount of energy in the $j$-th battery at $t$ (MW).

$R_{j}^{\max }: \quad$ The maximum capacity of the $j$-th battery (MWh).

$R_{j}^{\text {min }}$ : The discharging infimum of the $j$-th battery. (MWh).

$\Delta R^{t}$ : $\quad$ Total energy increment of all batteries at time $t$ (MW).

$\Delta R_{j}^{t}: \quad$ The energy increment of the $j$-th battery time $t(\mathrm{MW})$.

$\beta_{j}^{\text {High }}$ : $\quad$ Charging/discharging high rate of the $j$-th battery (MW).

$\beta_{j}^{\text {Norm }}$ : $\quad$ Charging/discharging normal rate of the $j$-th battery (MW).

$\beta_{j}^{\text {safe }}$ : $\quad$ Charging/discharging safe rate of the $j$-th battery (MW).

$C_{j}^{\text {High }}$ : $\quad$ Cost coefficient in high rate for the $j$-th battery (\$/MWh).

$C_{j}^{N o r m}: \quad$ Cost coefficient in normal rate for the $j$-th battery ( $\left.\$ / \mathrm{MWh}\right)$.

$C_{j}^{\text {Safe }}: \quad$ Cost coefficient in safe rate for the $j$-th battery( $\$ / \mathrm{MWh}$ )

$N^{t}: \quad$ The renewable energy available at time $t$ (MW).

This work was supported by the Australian Research Council (ARC) Linkage grant under Grant LP0991428.

C. Xiao, Prof. D. Sutanto \& K. M. Muttaqi are with School of Electrical, Computer and Telecommunications Engineering, Prof. M. Zhang is with School of Computer Science and Software Engineering, University of Wollongong, Wollongong, NSW 2522, Australia. (e-mail: chixinxiao@gmail.com, soetanto@uow.edu.au, kashem@uow.edu.au, minjie@uow.edu.au).

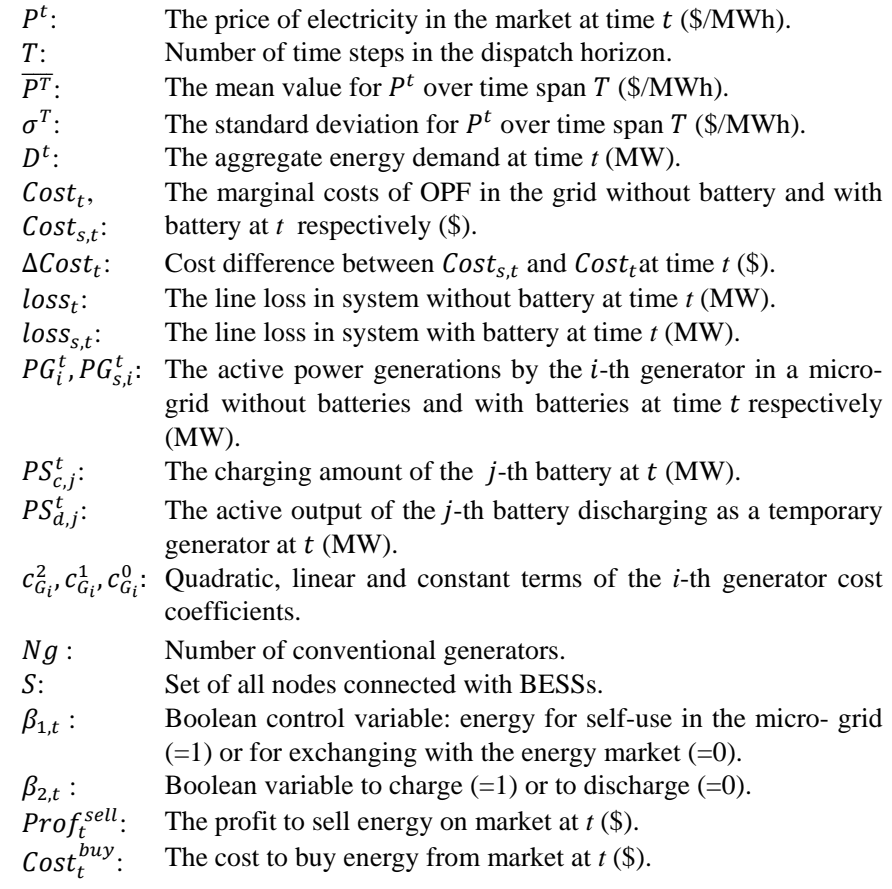

\section{INTRODUCTION}

THE distributed energy resources (DERs), such as the small-scale renewable energy resources (RES) and the energy storage system in the form of a Battery Energy Storage System (BESS)[1], can be aggregated to form a Virtual Power Plant (VPP) [2] and belong to an aggregator that can buy and sell electricity from and to the market as one big organization. In this paper, it is assumed that each VPP must have at least one BESS, and the power trading can be processed in a gridconnected environment via a wholesale market (WM).

The aggregated DERs as a VPP then communicates to the VPP control centre (VCC) the information on the available DERS in the VPP, such as their available active and reactive powers, their capacities and their operating parameters. At the same time, the VCC receives the information of the marginal cost of generation [3], the current values of system loads and their future prediction from the grid and the VCC also receives the spot price of the electricity and its future prediction from the WM. Based on the information that the VCC receives, the VCC sends the most optimum schedules of the BESSs in the DERs to the VPP at each time instance to minimize the cost and to maintain the generation and load balance. The VCC then decides on the amount of energy to buy or sell (dependent on the available capacity and energy in each component in 
VPP) and sends the WM the contracts and the offers to buy and sell electricity.

Fig. 1(a) shows a simplified power grid initially used in this paper, where the DERs are aggregated as the VPP, and how the VPP are physically connected to a simplified grid at two nodes. Fig. 1(b) shows symbolically how the VPP communicates with the VCC and the grid.

The VCC uses the Stochastic Optimization (SO) [4][5] method to achieve the maximum revenue over a time-span. The main challenge to solve such a SO problem [4] is to find an optimal lookahead plan (i.e., predictive control) among all possible-state trajectories, considering the uncertainties in the prediction and the contingencies in the grid. In each time slice, the VCC controls the charging/discharging of the battery storages by responding to the real-time spot price, while referring to the rolling price prediction; secondly, the information of the marginal cost of generation needs to be updated corresponding to the instant actions of the battery storages.

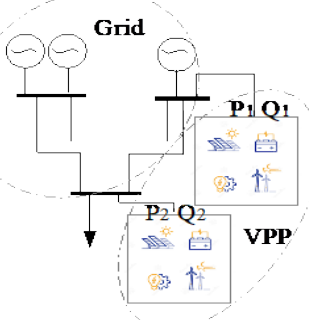

(a)

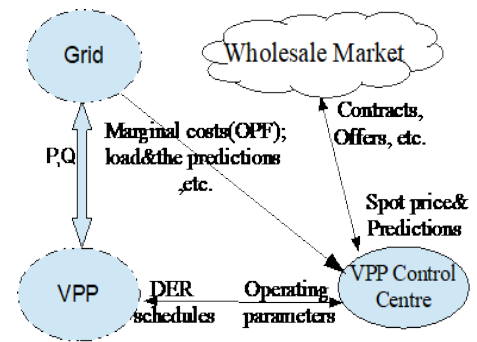

(b)
Fig.1 The Model of Controlling VPP: (a) The Topology of the Decentralized Dispatch with VPP (located at two nodes in the power grid), (b) Communication Scenario.

There is a vast literature in sequential SO-methods, such as the Markov Decision Processes (MDPs) [5], the backward dynamic programming algorithm (BDPA) [6], the economic model predictive control (EMPC) [7], etc., that proposes to blend modelling with the design of lookahead control laws (i.e. policies) [4]. When using the scenario tree approach, that considers all possible-state trajectories, the lookahead control law must determine all uncertainties for an optimal scenario (i.e. the optimal lookahead plan). However, these SO-methods may become computationally intractable when the problemscale increases. For a better universal and efficient sequential SO-method, the modelling needs to be separated from the design of the policies [4][5].

According to the compiler principle [ 8 ][9] used in computer science, a programming language is used as the formal language to perform various kinds of tasks and is programmed in the software level (i.e., the universal abstraction level) and the corresponding instruction flow (in which each instruction belongs the finite instruction set) is then executed in the hardware level (i.e., machine language level).

This motivates us to develop a three-layer model to solve the sequential SO problem to tackle the real-time control of charging/discharging of the battery storages: 1) the finite control-symbol set layer (i.e., machine language level), where the purpose of this layer is to create a set of control symbols and define the semantic meaning for each control symbol by quantifying each control symbol with a corresponding charging/discharging rate and cost of the given BESS; 2) the formal grammar layer, where the purpose of this layer is to define the syntax for the formal language [9], i.e., to provide a set of regular expressions to form all correct combinations based on the finite control symbol set, furthermore, a corresponding non-deterministic finite automaton (NFA) [9] [10] can be constructed to recognize all these regular expressions (i.e., the given formal language); and 3) the language generation layer (i.e., the software level), where the purpose of this layer is to discover the real-time profitable pattern from the spot price and its rolling prediction (i.e., the linguistic sources to produce the defined language) by using an intelligent algorithm (e.g., an extraneous learning algorithm) and to translate the found pattern into a sequence of control symbols (i.e., a sample of the given language) to drive the BESS.

Due to the continuous charging or discharging actions of the battery storages, the grid power balance based on OPF [11][12] needs to be updated in time corresponding to every operation of the battery storages [13][14]. The proposed OPF formulation is given in Section III-B.

From the discussion above, this paper proposes a novel real-time stochastic multi-period management strategy of a virtual power plant (VPP) using the proposed three-layer language protocol to simultaneously (i) minimize the operation cost of a smart grid containing distributed generation energy storage using the multi-period OPF taking into account not only the network and generator constraints but also the interaction with the WM, and (ii) maximize the profit when the VPP is operating in the electricity market, where each sub-problem is solved dynamically considering a global planning and real-time response to the real-time market data. Initially, the global planning problem is decomposed into several real-time sub-problem sets. In each time slice, having obtained the global prediction of the power price from the market, the difference between the real-time price and the local operational cost is used to guide the operation of the storage to be: (i) a local generator, (ii) a local load, (iii) an energy buyer, (iv) an energy seller or (v) an energy hoarding pool. Hence, the supply and demand of the current subproblem are affected by the local operational cost, the realtime market price and its future prediction. To achieve a rapid and robust control strategy, several control symbols (associated with the charging and discharging of the BESS) with a recursive definition are fuzzified dynamically based on each 5-minute forecasted price in a 24-hour period from the WM. A corresponding non-deterministic finite automaton (NFA) is designed to translate the instructions in the control symbols from the VCC into special actions for the operation of the BESS. The optimization tools, e.g. OPF in MatPower and the buy-low-sell-high strategy are adopted to achieve the optimum real-time response to the real-time market data.

The contributions of this paper are:

1) The development of a novel real-time stochastic multiperiod management strategy of a virtual power plant (VPP) using a three-layer language protocol to solve the 
sequential SO problem to control in real time the charging/discharging of the battery storages;

2) The formalization of the mapping of the BESS charging and discharging with the forecasted prices into several finite states and finite intervals respectively to reduce the large possible states of the stochastic optimization, and to ensure the strict feasibility of the optimal trajectory;

3) The formalization of the non-deterministic finite automaton (NFA) that includes BESS, the instant spot price and the price prediction, to implement a recursive and convenient algorithm for spot price trading and the tariff arbitrage over a time-span;

4) The determination of the timing and the amount of the charging or discharging of the BESS by the intelligence of the VCC to provide the energy buffer to the intermittent and fluctuating sources as it receives the variation of the electricity market price;

5) The inclusion of a dynamic OPF model that can ensure the balance between the generation and the load plus losses, the guarantee that the constraints in the power system can be met and the optimization of the operation cost of the grid and the VPP.

The rest of the paper is organized as follows. Section III outlines the proposed problem in a mathematical manner. The proposed approach is described in Section IV. In Section V, the simulation results and analysis are presented. Finally, in Section VI, the conclusion and future work are provided.

\section{BASIC BACKGROUNDS AND PROBLEM FORMULATION}

The first test system used in this paper is shown in Fig. 1(a) and the received and the sending communications of the VCC is shown in Fig. 1(b). Fig. 1(a) shows a grid with $n$ buses consisting of $\mathrm{Ng}$ conventional generation $\left(P G_{i}^{t}\right)$, and load demand $\left(D^{t}\right)$ and a VPP with renewables $\left(N^{t}\right)$ and BESS $\left(R^{t}\right)$. In the proposed VPP arrangement, the grid can purchase or sell power to the VPP based on the determination made by the VCC depending on the current spot price and its future prediction to maximise profits for the VPP and to minimize the generation cost of the grid.

\section{A. Traditional Multi-Period OPF}

(1) gives a common quadratic cost function for a conventional generator and (1a) defines the total optimal generation cost at time $t$.

$$
\begin{aligned}
& f\left(P G_{i}^{t}\right)=c_{G_{i}}^{2} \cdot\left(P G_{i}^{t}\right)^{2}+c_{G_{i}}^{1} \cdot P G_{i}^{t}+c_{G_{i}}^{0} \\
& \text { Cost }_{t}=\text { Minimize }\left(\sum_{i=1}^{N g} f\left(P G_{i}^{t}\right)\right) \\
& \text { Reference Cost }=\sum_{t=1}^{T} \operatorname{Cost}_{t}
\end{aligned}
$$

Both (1) and (1a) are subject to:

$$
\begin{aligned}
& \sum_{i=1}^{N g} P G_{i}^{t}=D^{t}+\operatorname{loss}_{t} \quad, i \in[1, N g] \\
& P G_{\text {min }, i} \leq P G_{i}^{t} \leq P G_{\text {max }, i}, i \in[1, N g] \\
& Q G_{\text {min }, i} \leq Q G_{i}^{t} \leq Q G_{\text {max }, i}, i \in[1, N g] \\
& V_{\text {min }, k} \leq V_{k}^{t} \leq V_{\text {max }, k}, k \in[1, n]
\end{aligned}
$$

\section{B. The Proposed Multi-Period OPF with VPP}

This case includes such factors as renewables $\left(N^{t}\right)$, BESS $\left(R^{t}\right)$ and energy transaction to the traditional OPF. Hence, (1a) and (1b) can be revised to (2) and (2a).
$\operatorname{Cost}_{s, t}=\operatorname{Minimize}\left[\sum_{i=1}^{N g} f\left(P G_{s, i}^{t}\right)+\beta_{1, t} \cdot \sum_{j=1}^{S} f\left(P S_{d, j}^{t}\right)\right]$ where $f\left(P G_{s, i}^{t}\right)$ and $f\left(P S_{d, j}^{t}\right)$ now includes the impact of having $S$ numbers of BESSs either charging or discharging. (1c) can now rewritten as (2a) be subject to (1d), (1e), (1f):

$$
\sum_{i=1}^{N g} P G_{s, i}^{t}+N^{t}=D^{t}+\operatorname{loss}_{s, t}+\beta_{1, t} \cdot \Delta R^{t}
$$

(2a) ensures that the generation by all generation (including renewables and batteries) is balanced by the load and losses.

The proposed method can be described in two steps: First, based on the instant price and the prediction, a real-time synchronized movement for all BESSs are decided, either to charge or to discharge.

$$
\beta_{2, t}=\left\{\begin{array}{l}
1, \quad \text { if } P^{t} \leq \overline{P^{T}} \text {, charging } \\
0, \quad \text { if } P^{t}>\bar{P}^{T}, \text { discharging }
\end{array}\right.
$$

(2b) determines whether the battery is charging or discharging, $\beta_{2, t}$, based on the comparison of the current price, $\left(P^{t}\right)$, and the average price, $\left(\overline{P^{T}}\right)$.

$\Delta R_{j}^{t}=R_{j}^{t}-R_{j}^{t-1}=\left\{\begin{array}{l}P S_{c, j}^{t}, \quad \text { for } \& \beta_{2, t}=1, \text { charging } \\ P S_{d, j}^{t}, \quad \text { for } \beta_{2, t}=0, \text { discharging }\end{array},(1 \leq j \leq S)\right.$

(2c) determines the individual battery energy available $\left(\Delta R_{j}^{t}\right)$ for charging or discharging:

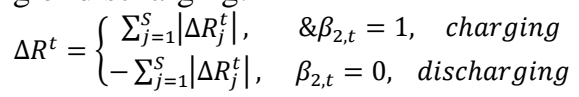

(2d) determines the total battery energy available $\left(\Delta R^{t}\right)$ for charging or discharging.

The profit and cost can be calculated from (2g) and 2(h).

$$
\text { temp }=\text { Cost }_{t}-\left[\sum_{i=1}^{N g} f\left(P G_{s, i}^{t}\right)+\sum_{j=1}^{S} f\left(P S_{d, j}^{t}\right)\right]
$$

(2e) shows the difference, temp , between the reference cost and the total cost of the battery energy whether charging or discharging.

$$
\beta_{1, t}=\left\{\begin{array}{l}
0, \quad\left(\mid \text { temp }\left|-P^{t} \cdot\right| \Delta R^{t} \mid\right) \leq 0 \text { and } \beta_{2, t}=0 \\
1, \quad\left(\mid \text { temp }\left|-P^{t} \cdot\right| \Delta R^{t} \mid\right)>0 \text { and } \beta_{2, t}=0 \\
1, \quad\left(\mid \text { temp }\left|-P^{t} \cdot\right| \Delta R^{t} \mid\right) \leq 0 \text { and } \beta_{2, t}=1 \\
0,\left(\mid \text { temp }\left|-P^{t} \cdot\right| \Delta R^{t} \mid\right)>0 \text { and } \beta_{2, t}=1
\end{array}\right.
$$

(2f) uses temp to decide on the purposes of batteries for charging and discharging as shown in Table I, e.g., to obtain profit, the batteries are discharged either as energy sellers $\left(\beta_{2, t}=0\right.$ and $\left.\beta_{1, t}=0\right)$ or as temporary generators $\left(\beta_{2, t}=0\right.$ and $\left.\beta_{1, t}=1\right)$; similarly the batteries are charged either as energy buyers $\left(\beta_{2, t}=1\right.$ and $\left.\beta_{1, t}=0\right)$ or as temporary loads $\left(\beta_{2, t}=1\right.$ and $\left.\beta_{1, t}=1\right)$. When the batteries are used as energy buyers or energy sellers, the energies are bought or sold to the market and will therefore not affect the local grid, however when the batteries are used as temporary generators or temporary loads, the energies must be supplied or drawn from the local grid.

Table I. Energy Storage Working Character in Different Situations

\begin{tabular}{c|cc}
\hline \hline & $\beta_{1, t}=0$ & $\beta_{1, t}=1$ \\
\hline$\beta_{2, t}=0$ & Energy Seller & Temporary Generator \\
$\beta_{2, t}=1$ & Energy Buyer & Temporary Load \\
\hline \hline
\end{tabular}

Eq. (2g) determines the profit based on the decision of the use of the batteries as energy sellers.

$$
\operatorname{Cost}_{t}^{b u y}=\left\{\begin{array}{cc}
0 & , \beta_{2, t}=0 \text { and } \beta_{1, t}=0 \\
P^{t} \cdot\left|\Delta R^{t}\right|, \beta_{2, t}=1 \text { and } \beta_{1, t}=0
\end{array}\right.
$$

Eq. ( $2 \mathrm{~h}$ ) determines the cost based on the decision of the use of the batteries as energy buyers.

\section{Optimization Objects}

There are two main objectives in this paper: one is to 
maximize the revenue of VPP over $[1, T]$ on the market, which can be described as (3)

$$
\text { Market Revenue }=\text { Maximize } \sum_{t=1}^{T}\left(\operatorname{Prof}_{t}^{\text {sell }}-\text { Cost }_{t}^{\text {buy }}\right)
$$

The other is to minimize the operational cost of the grid over $[1, T]$ based on (4),

$$
\text { Total Cost }=\text { Minimize } \sum_{t=1}^{T} \text { Cost }_{s, t}
$$

In addition, some auxiliary definitions are given as follows,

$$
\Delta \text { Cost }_{t}=\text { Cost }_{t}-\text { Cost }_{s, t}
$$

where $\Delta$ Cost $_{t}$ represents the contribution of VPP on the reference cost at time slice $t$.

The total revenue over $[1, T]$ can be expressed as (5a),

Total revenue $=$ Market Revenue + Maximize $\left(\sum_{t=1}^{T} \Delta \operatorname{Cost}_{t}\right)$

The real cost over $[1, T]$ can be expressed as (5a),

$$
\text { Real } \text { Cost }=\text { Reference cost } \text {-Total revenue }
$$

\section{DATA-DriVEN DETERMINISTIC LOOKAHEAD APPROACH}

In the proposed strategy, each BESS in VPP must not only consider the optimality of the energy self-use for the local grid but also pursue to obtain more revenue, which are triggered by the information extracted from the real-time price data in each single period.

\section{A. The Finite Control-Symbol Set of BESS}

The dynamic decision for charging or discharging is to use 'buy low and sell high' strategy [15], causing the control actions to respond to the real time price to benefit over a time span rather than a single-time point. A precise prediction [16] and regular updating based on historical data is necessary [17].

Based on the prediction, the forecasted price (e.g., each 24hour prediction can be divided into four levels from bottom to top: there are lower price area, $P^{t} \in C_{2}=\left\{P^{t} \mid P^{t} \leq \overline{P^{T}}-\sigma^{T}\right\}$, low price area, $P^{t} \in C_{1}=\left\{P^{t} \mid \overline{P^{T}}-\sigma^{T}<P^{t} \leq \overline{P^{T}}\right\}$, high price area, $P^{t} \in$ $D_{1}=\left\{P^{t} \mid \overline{P^{T}}<P^{t} \leq \overline{P^{T}}+\sigma^{T}\right\}$, and higher price area, $P^{t} \in D_{2}=$ $\left\{P^{t} \mid \overline{P^{T}}+\sigma^{T} \leq P^{t}\right\}$, respectively as shown Fig. 2 .

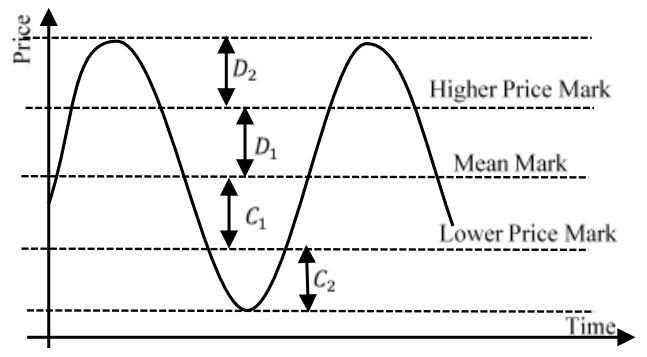

Fig. 2. Predicted Price Curve in 4 levels

When $P^{t}$ is within $C_{1}$ or $C_{2}$ area, it is preferable to charge the BESS and hoard the energy, rather than using the energy of the BESS to supply the load, with the intention of selling the stored energy to the market when the price $P^{t}$ is within the $D_{1}$ or $D_{2}$ area to obtain profit. Moreover, when the price is in $C_{2}$, the battery will be charged at a higher charging rate to store more energy with the expectation of selling more energy in $D_{2}$ to generate more revenue. In general, the proposed strategy is to maximize the sum of all the differences over time interval $[1, T]$, as shown in (5), to optimize the profit and to promote the effective use of the local energy sources and the BESS.

\section{1) Dynamic Charging/Discharging Rate}

When the state of charge (SoC) exceeds the upper or lower limit (e.g., in this paper the range is set between $10 \%$ to 90\%) of the SoC of the battery, the discharging rate should be reduced to extend the time that the battery is available when the SoC is between $10 \%$ and $20 \%$, and similarly the charging rate should be reduced to extend the time that the storage is available when the SoC is between $80 \%$ to $90 \%$.

The control logic in Table II is adopted to explain the idea of the dynamic charging and discharging rate. For example, when the current price is above the mean mark shown in Fig. 2, the storages should be discharging at the highest charging rate $\beta_{j}^{\text {High }}$ when $P^{t} \in D_{2}$ or at their normal rate $\beta_{j}^{\text {Norm }}$ when $P^{t} \in D_{1}$. Their cost rates are $C_{j}^{\text {High }}$ and $C_{j}^{\text {Norm }}$ respectively. Further, when

\begin{tabular}{|c|c|c|c|}
\hline \multicolumn{2}{|c|}{ Conditions } & $\begin{array}{c}\text { Charging } \\
\text { Rate\&Unit Cost }\end{array}$ & $\begin{array}{c}\text { Discharging } \\
\text { Rate \&Unit Cost }\end{array}$ \\
\hline $\begin{array}{c}R_{j}^{t} \in[0.8 * \\
R_{j}^{\max }, 0.9 * \\
\left.\quad R_{j}^{\max }\right]\end{array}$ & $\begin{array}{l}P^{t} \in D_{2} \\
P^{t} \in D_{1} \\
P^{t} \in C_{1} \\
P^{t} \in C_{2}\end{array}$ & $\begin{array}{c}- \\
\beta_{j}^{\text {Safe }} \& C_{j}^{\text {Safe }} \\
\beta_{j}^{\text {Safe }} \& C_{j}^{\text {Safe }}\end{array}$ & $\begin{array}{c}\beta_{j}^{\text {High }} \& C_{j}^{\text {High }} \\
\beta_{j}^{\text {Norm }} \& C_{j}^{\text {Norm }} \\
-\end{array}$ \\
\hline $\begin{array}{l}R_{j}^{t} \\
\in[0.1 \\
* R_{j}^{\max }, 0.2 \\
\left.* R_{j}^{\max }\right]\end{array}$ & $\begin{array}{l}P^{t} \in D_{2} \\
P^{t} \in D_{1} \\
P^{t} \in C_{1} \\
P^{t} \in C_{2}\end{array}$ & $\begin{array}{c}- \\
\beta_{j}^{\text {Norm }} \& C_{j}^{\text {Norm }} \\
\beta_{j}^{\text {High }} \& C_{j}^{\text {High }}\end{array}$ & $\begin{array}{l}\beta_{j}^{\text {Safe }} \& C_{j}^{\text {Safe }} \\
\beta_{j}^{\text {Safe }} \& C_{j}^{\text {Safe }}\end{array}$ \\
\hline $\begin{array}{l}R_{j}^{t} \\
\in[0.2 \\
* R_{j}^{\max }, 0.8 \\
\left.* R_{j}^{\text {max }}\right]\end{array}$ & $\begin{array}{l}P^{t} \in D_{2} \\
P^{t} \in D_{1} \\
P^{t} \in C_{1} \\
P^{t} \in C_{2}\end{array}$ & $\begin{array}{c}- \\
\beta_{j}^{\text {Norm }} \& C_{j}^{\text {Norm }} \\
\beta_{j}^{\text {High }} \& C_{j}^{\text {High }}\end{array}$ & $\begin{array}{c}\beta_{j}^{\text {High }} \& C_{j}^{\text {High }} \\
\beta_{j}^{\text {Norm }} \& C_{j}^{\text {Norm }} \\
-\end{array}$ \\
\hline
\end{tabular}
the SoC is over or below the upper or lower limit, no further charging or discharging is allowed.

Table II BESS Operating Logic Based on Data-Driven

The algorithm in Fig. 3(a) provides the working details while discharging and the one shown in Fig. 3(b) explains the charging processing.

\section{2) The Formal Grammar for the Data-driven Control}

For the real-time data-driven control, it is an important step to generalize all the dynamic storage-actions by a recursive method. Thus, a regular grammar [18] is defined in (6)-(6c), which aims to encode the forecasted price data, in each 24period, into action-symbols corresponding to the four price levels as shown in Fig. 2.

$$
\begin{aligned}
& S \rightarrow T S \mid F \\
& T \rightarrow C T|D T| C \mid D \\
& C \rightarrow C_{1} \mid C_{2} \\
& D \rightarrow D_{1} \mid D_{2}
\end{aligned}
$$

where ' $S$ ' represents the start symbol (i.e., nonterminal symbol) [8]-[10] of the set of finite control symbols (i.e., terminal symbols [8]-[10] such as ' $C_{1}{ }^{\prime},{ }^{\prime} C_{2},{ }^{\prime},{ }_{1}{ }^{\prime},{ }^{\prime} D_{2}$ ' and ' $F$ ') for the battery storages. It can be defined recursively as a subsymbol group ' $T$ ' followed by ' $S$ ' itself or a final symbol ' $F$ '. As given in (6a), for any ' $T$ ', its follow-up symbols may begin at a charging symbol, ' $C$ ', or a discharging one, ' $D$ ', attached with a recursive ' $T$ ' set. (6b) and (6c) mean the symbol ' $C$ ' or ' $D$ ' may decompose further into its terminal symbols for more precise actions, e.g., ' $C_{1}{ }^{\prime},{ }^{\prime} C_{2}$ ', ' $D_{1}{ }^{\prime}$, ' $D_{2}$ ' as shown in Fig.2.

Then, the language based on the grammar given in (6) (6c), (i.e., all strings only consist of terminal symbols and are derived from the start symbol, S), is an infinite set, and as shown in (6d), 


$$
\left\{\left(C_{1}{ }^{i 1} C_{2}{ }^{i 2} D_{1}{ }^{i 3} D_{2}{ }^{i 4} F^{i}\right)^{*} \mid i 1, i 2, i 3, i 4 \geq 0 \& i=0 \text { or } 1\right\}
$$
where $C_{1}{ }^{i 1}$ is ' $C_{1}$ ' repeated $i 1$ times, and so on, the operator '*' represents the Kleene closure [9], which means 'zero or more'. For example, the string ' $C_{2} C_{2} D_{1} F$ ' can be derived by starting with ' $S$ ' with a series of substituting of the regular expressions among (6)-(6c). The deriving process can be expressed briefly by using symbols: $S \stackrel{(6)}{\Longrightarrow} T S \stackrel{(6 a)}{\Longrightarrow} C T S \stackrel{(6 b)}{\Longrightarrow} C_{2} T S \stackrel{(6 a)}{\Longrightarrow} C_{2} C T S \stackrel{(6 b)}{\Longrightarrow} C_{2} C_{2} T S \stackrel{(6 a)}{\Longrightarrow}$ $C_{2} C_{2} D S \stackrel{(6 c)}{\Longrightarrow} C_{2} C_{2} D_{1} S \stackrel{(6)}{\Rightarrow} C_{2} C_{2} D_{1} F$.
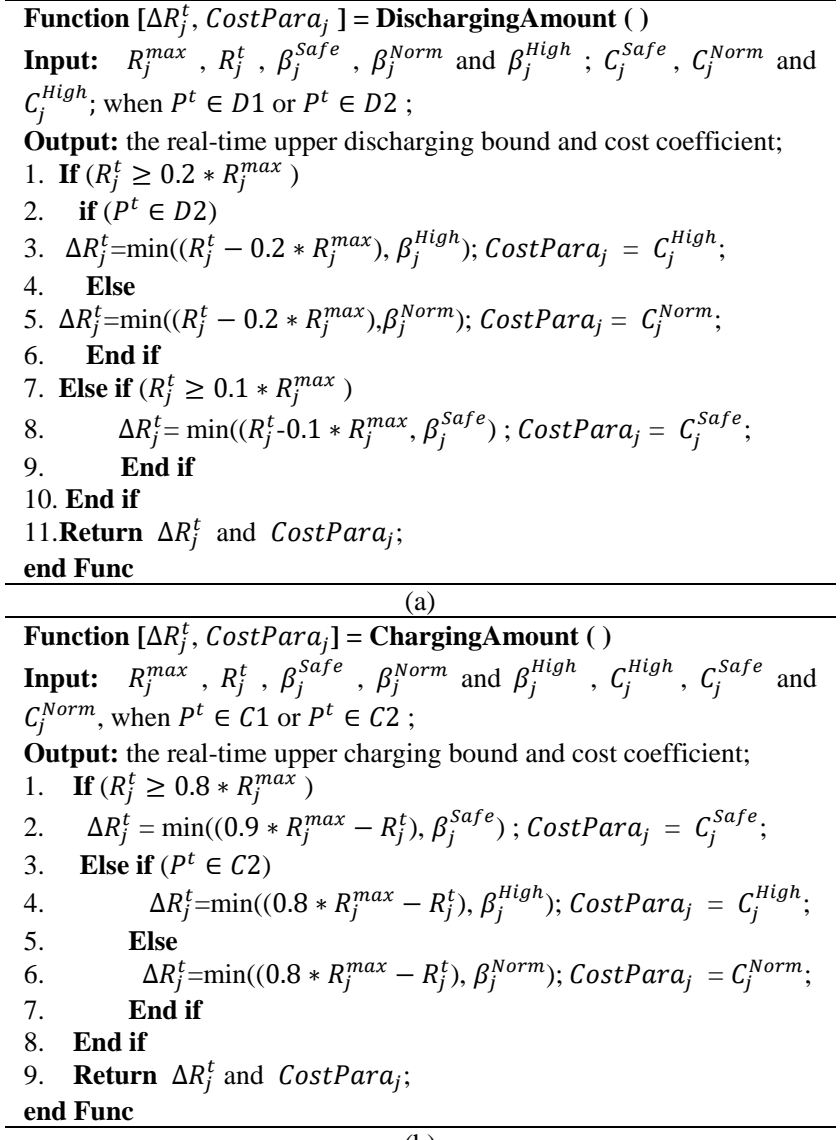

Fig. 3 Preparation for real-time charging or discharging (a) Discharging amount strategy, (b) Charging amount strategy

Hence, via the grammar, the successive real-time price data can be translated into a series of instructions sent from the VCC to the distributed battery storages (i.e., a programmed sequence of instructions). That is, the instruction sequence is a string pattern consisting of such control symbols as ' $C_{1}$ ',' $C_{2}$,', $D_{1}$ ',' $D_{2}$ ' and ended with the symbol ' $F$ '. For example, suppose the real-time price is $P^{t}=\$ 49 / \mathrm{MWh}$, based on the prediction over the whole time span (assume the average price is $\overline{P^{T}}=\$ 55 / \mathrm{MWh}$ and the standard deviation is $\sigma^{T}=10$ ), the battery storage must receive a control symbol, ' $C_{1}$ ' (i.e., $\overline{P^{T}}-$ $\sigma^{T}<49<\overline{P^{T}}$ ), rather than a number, '49'; Assume the battery storage receives a control-symbol string like ' $C_{1} D_{1} D_{2} D_{1} \ldots$ ', it can be decoded directly to a set of storage actions, i.e., charging, discharging, fast discharging, discharging... If necessary, the noise interference around $\overline{P^{T}}, \sigma^{T}$ and the dynamic update on the prediction may be considered, but this will be out of the scope of the paper, and therefore is not considered here.

\section{B. State Transition of BESS}

On the BESS side, corresponding to the recursive definition for the control symbols, the dynamic energy volume of each battery storage can also be reflected by a serial sequence consisting of finite states: charging state, $S_{c}$, discharging state, $S_{d}$, empty state, $S_{E}$, and full state, $S_{F}$ ) rather than the conventional approaches that consider every possible continuous state, which can often cause the computational complexity to become intractable.

The whole state transition procedure is shown in Fig. 4 and is a non-deterministic finite automaton (NFA) in accordance to the regular grammar (in Section IV-A-2), which is easier to implement by software or hardware and is defined as follows,

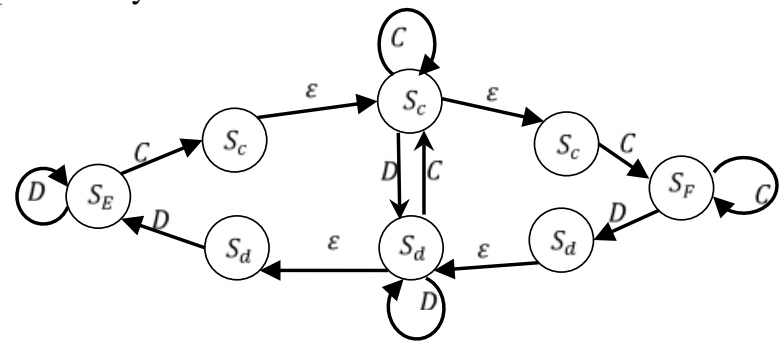

Fig. 4. Storage State diagram for Dynamic Transition

Definition: a 5-tuple $M=\left(Q, \sum, \delta, q_{0}, F\right)$, consisting of: a finite set of states $Q=\left\{S_{c}, S_{d}, S_{E}, S_{F}\right\}$; a finite set of input symbols $\sum=$ $\left\{C_{1}, C_{2}, D_{1}, D_{2}, \varepsilon\right\}, \varepsilon$ is an empty symbol; a start state $q_{0} \in Q$; a set of accept states $F \subseteq Q$; a transition function, $\delta: Q \times \sum \rightarrow Q$, is defined by Table III.

Table III Battery State Transition Rule for Programming

\begin{tabular}{c|ccccc}
\hline \hline & $C_{1}$ & $C_{2}$ & $D_{1}$ & $D_{2}$ & $\varepsilon$ \\
\hline$S_{E}$ & $S_{c}$ & $S_{c}$ & $S_{E}$ & $S_{E}$ & $S_{E}$ \\
$S_{c}$ & $\left\{S_{c}, S_{F}\right\}$ & $\left\{S_{c}, S_{F}\right\}$ & $S_{d}$ & $S_{d}$ & $S_{c}$ \\
$S_{d}$ & $S_{c}$ & $S_{c}$ & $\left\{S_{d}, S_{E}\right\}$ & $\left\{S_{d}, S_{E}\right\}$ & $S_{d}$ \\
$\left\{S_{c}, S_{F}\right\}$ & $S_{F}$ & $S_{F}$ & $S_{d}$ & $S_{d}$ & $\left\{S_{c}, S_{F}\right\}$ \\
$\left\{S_{d}, S_{E}\right\}$ & $S_{c}$ & $S_{c}$ & $S_{E}$ & $S_{E}$ & $\left\{S_{d}, S_{E}\right\}$ \\
$S_{F}$ & $S_{F}$ & $S_{F}$ & $S_{d}$ & $S_{d}$ & $S_{F}$ \\
\hline \hline
\end{tabular}

In Fig.4, ' $C^{\prime}$ may be substituted by one control symbol, either $C_{1}$ or $C_{2}$, just as defined in (6c), similarly, ' $D$ ' appears as $D_{1}$ or $D_{2}$. When receiving a charging/discharging instruction ' $C$ ' or ' $D$ ' (as shown in the first row of Table III) at time slice $t$, a battery needs a state-transfer from its current state (the most left column in Table III) into its next state based on the state transition rule listed in Table III. Corresponding to the new state of the battery, the energy of the battery needs updating following the rules given in (7).

$$
\left\{\begin{array}{cc}
S_{E}: & R_{j}^{t+1}=R_{j}^{t}=R_{j}^{\text {min }} ; \\
S_{c}: & R_{j}^{t+1}=R_{j}^{t}+\Delta R_{j}^{t} ; \\
S_{d}: & R_{j}^{t+1}=R_{j}^{t}-\left(\Delta R_{j}^{t} \text { or } P S_{d, j}^{t}\right) ; \\
S_{F}: & R_{j}^{t+1}=R_{j}^{t}=R_{j}^{\text {max }} ;
\end{array}\right.
$$

where the first expression and the last one in (7) respectively correspond to two idle situations of batteries when the energy is not changed. As shown in Fig. 4, one idle situation is at the maximum energy state $S_{F}$ while receiving continuous charging-instructions, the other is at the minimum energy state $S_{E}$ while receiving continuous discharging-instructions. Otherwise, at time slice $t$, when the $j^{\text {th }}$ battery transfers into a new charging state $S_{c}$, its hoarding energy should be updated by $R_{j}^{t+1}=R_{j}^{t}+\Delta R_{j}^{t} \quad$ or by $R_{j}^{t+1}=R_{j}^{t}-\left(\Delta R_{j}^{t}\right.$ or $\left.P S_{d, j}^{t}\right)$ in the discharging state $S_{d}$., where $\Delta R_{j}^{t}$ and $P S_{d, j}^{t}$ are determined by 
using the methods as shown in Fig. 3.

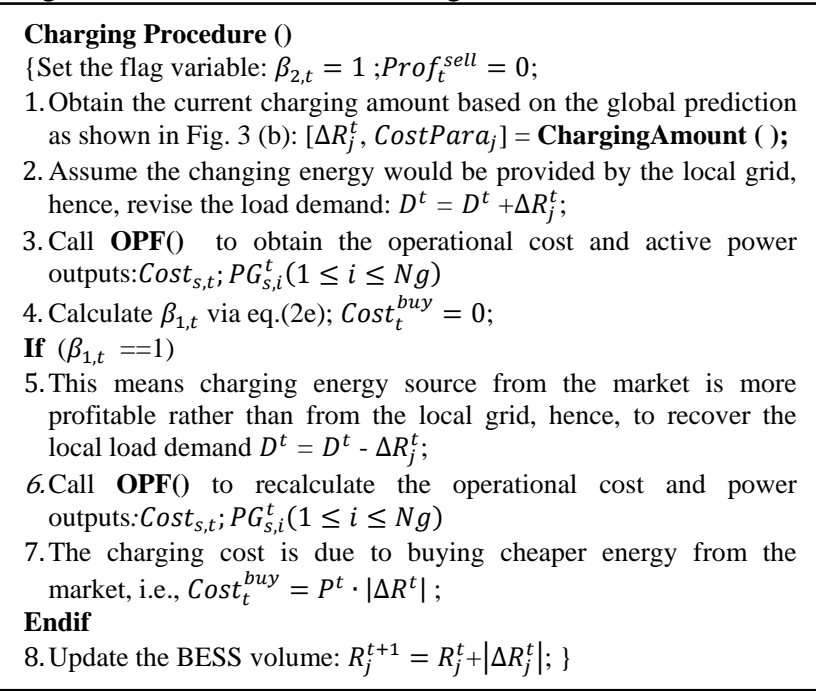

(a)

\section{Discharging Procedure ()}

\{Set the flag variable: $\beta_{2, t}=0 ; \operatorname{Cost}_{t}^{\text {buy }}=0$;

1. Obtain the current discharging amount based on the global prediction as shown in Fig. 3 (a): $\left[\Delta R_{j}^{t}, \operatorname{CostPara}_{j}\right]=$ DischargingAmount( );

2. The BESS would act as temporary generators, thus, to increase temporary generators into generator list with maximum capacity $\Delta R_{j}^{t}$.

3. Call $\boldsymbol{O P F} 0$ to obtain the operational cost and active power outputs: $\operatorname{Cost}_{s, t} ; P G_{s, i}^{t}, P S_{d, j}^{t}(1 \leq i \leq N g, 1 \leq j \leq S)$

4. Calculate $\beta_{1, t}$ via eq.(2e);

5. Update the BESS volume: $R_{j}^{t+1}=R_{j}^{t}-P S_{d, j}^{t}$; Prof $f_{t}^{\text {sell }}=0$;

If $\left(\beta_{1, t}==1\right)$

6. This means to sell discharging energy to the market is more profitable rather than to support the local grid, hence, it is necessary to delete the temp generators from the list and to recover original setting;

7. Recall $\boldsymbol{O P F}(0$ to obtain the operational cost and active power outputs: $\operatorname{Cost}_{s, t} ; P G_{s, i}^{t}(1 \leq i \leq N g)$

8. The revenue to sell energy on the market is: $\operatorname{Prof}_{t}^{\text {sell }}=P^{t} \cdot\left|\Delta R^{t}\right|$; 9. Update the BESS volume: $R_{j}^{t+1}=R_{j}^{t}-\left|\Delta R_{j}^{t}\right|$; endif $\}$

(b)

\section{Main procedure() \\ 1.Initialization;}

Do \{

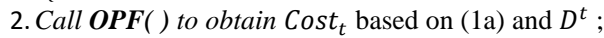

3. Run Programming (if $t=1$ ) or Updating (if $1<t<=T$ ) the lookahead control policy based on the formal grammar in Section IV A-2 according to the real-time price and its rolling prediction;

If (the real-time instruction is $\left(C_{1} \| C_{2}\right)$

4. Charging Procedure (); \% as shown in Fig. 5(a)

else

5. Discharging Procedure (); \% as shown in Fig. 5(b)

Endif

6. Calculate $\Delta \operatorname{Cost}_{t}$ and record down the real-time decisions: $\operatorname{Cost}_{s, t}$, Cost $_{t}$, Prof $_{t}^{\text {sell }}$, Cost $_{t}^{\text {buy }}$

7. To transfer to the next time slice $t=t+1$;

\}Until $(t>T)$

8. Assess the final results over [1, T], based on (3) , (4) and (5)

End Main

(c)

Fig.5 The data-driven control algorithm used in VCC: (a) Charging source control, (b) Discharging purpose control, (c) The main control procedure

\section{Dynamic use of BESS}

So far, the VCC at time $t$ has decided whether to charge or discharge and how much energy can be used in charging or discharging.

However, the BESS in this paper not only plays as a storage but also as an energy router [19]. The distributed energy resources (DERs) are connected to local electrical grids and controlled by cloud technologies [20] (the details are out of the scope of this paper). When the local supply exceeds the local demand, the surplus energy can be stored into the BESS. When the energy price is profitable, the BESS can discharge energy into the grid or buy energy from the grid for charging (i.e., inter-regional trading). At the time of local energy deficit, the BESS provides the insufficient amount of energy (i.e. compensating as local supply). Thus, the operation of the battery storages can be: (i) a local generator, (ii) a local load, (iii) an energy buyer, (iv) an energy seller, or (v) an idle state. Before making the decision about the mode of operation it should be, an auxiliary calculation, via a traditional OPF method shown in Section III-A (1)-(1f) (e.g. Newton-Raphson method), is adopted here and the details are shown in Fig. 5 and discussed below:

1) For the charging case of the BESS as a local load or energy buyer: The real charging amount for each battery can be obtained using the pseudo code as given in Fig. 5(a).

2) For the discharging case of the BESS as a local generator or an energy seller: the storage plays as a temporary generator to contribute $\Delta R^{t}$ energy in total to the local grid, or an energy seller to sell the same quantity of energy to the market for profit, the discharging amount has given in Fig. 5(b).

Fig.5 (c) shows the pseudocode of the main control procedure running on the VCC side. In the initialization (the $1^{\text {st }}$ step), the parameters of the three-layer model are prepared respectively: the finite control-symbol set layer (the hardware level), the formal grammar layer and the language generation layer (the software level or programming layer). In the $3^{\text {rd }}$ step, VCC samples the continuous price signal at regular time intervals (e.g. per 5-min or 30-min) and translates the samples to a sequential of control symbols by using the formal grammar in Section IV A-2, then triggers the NFA to change the state of the BESS by sending the instruction (i.e., the control symbol) to the BESS.

\section{SimUlation RESULtS AND ANALYSis}

In this part, two benchmark systems are adopted to illustrate the proposed strategy. The first case is shown in Fig. 6 adapted from the Tutorial Example System in [21]; the second one is shown in Fig. 7, which is the IEEE 14-bus system adapted from [22].

\section{A. The 3-bus system}

Fig. 6 shows the test system with two identical 200MW generators located at Bus 1 with different reserve costs denoted as Gen1 and Gen2, and one 500MW generator at Bus 2 denoted as Gen3. Only the linear coefficient are used in Eq. 
(1), whose values are set as: $c_{G_{1}}^{1}=75, c_{G_{2}}^{1}=90, c_{G_{3}}^{1}=120$, and the rest of the variables (the quadratic coefficients $\left(c_{G_{1}}^{2}, c_{G_{2}}^{2}, c_{G_{3}}^{2}\right)$ and the constants $\left(c_{G_{1}}^{0}, c_{G_{2}}^{0}, c_{G_{3}}^{0}\right)$ are set as 0 . The battery storage system at bus 3 is a 200MWh unit with 80 MW max charging/discharging rate as used in [21]. The price data and the load demand is scaled down from the historical data from September to October from the AEMO website [17].

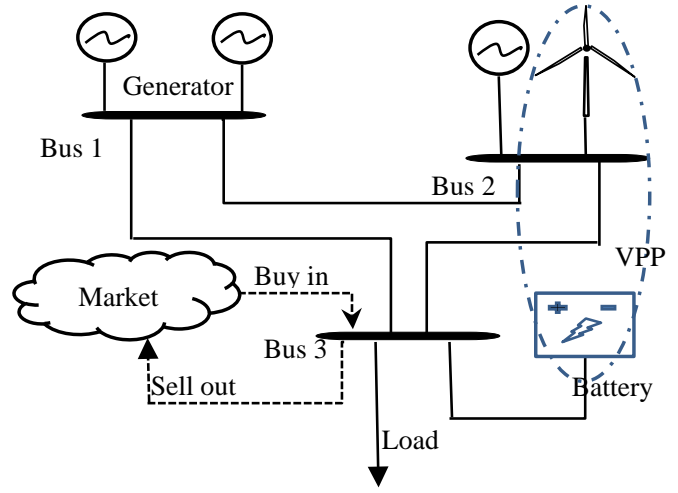

Fig. 6. A 3-bus test system with market and Battery [21]

The values of the variables mentioned in Table II are: $R_{j}^{\text {max }}=200 \mathrm{MWh}, \beta_{j}^{\text {High }}=80 \mathrm{MW}$, and $\beta_{j}^{\text {Norm }}=40 \mathrm{MW}, \beta_{j}^{\text {Safe }}=10 \mathrm{MW}$, and the cost rates $C_{j}^{\text {High }}, C_{j}^{\text {Norm }}$ and $C_{j}^{\text {Safe }}$, are $\$ 9 / \mathrm{MWh}$, \$5/MWh and $\$ 1 / \mathrm{MWh}$ respectively. The wind generator at Bus 2 (whose maximum output is $100 \mathrm{MW}$ ) is set as a stochastic case, where the output value is randomly varied at each time slice from 0 to $100 \mathrm{MW}$. The wind data $\left(N^{t}\right)$, the system load $\left(D^{t}\right)$ and the market price data $\left(P^{t}\right)$, are listed in the left three columns in Table IV. The output of the wind generator is treated as a negative load at bus 2 . The branch limits are set to be $300 \mathrm{MW}$ for line 1-2, 240MW for line 1-3 and 300MW for line 2-3. From the price data in Table 4, the average market price over one day, $\overline{P^{T}}$, is $\$ 139.87$ (based on which the decision of charging and discharging can be made as given in (2b), and the standard deviation $\sigma^{T}$ is $\$ 27.97$ (based on which the four levels of the price curve as shown in Fig. 2 can be obtained).

First, a conventional OPF is carried out by considering only the three conventional generators plus the wind generator over a 12-time interval. Second, the battery storage system with an initial energy of 40MW is added.

Initially, the battery is driven by a very simple control instruction, whether the batteries should be a temporary load or a temporary generator, and the battery can be fully charged or empty. The comparison of the results between the two cases is shown in Table IV. When $\Delta R_{j}^{t}$ is negative, it represents the discharging action of the battery storage, which supplies energy to the grid, and hence the battery storage acts a generator; and when it is positive, it represents the charging action of the battery storage, which draws energy from the grid and hence the battery storage acts as a load. The cumulative loss/benefit ends with a positive value that means the use of the battery storage system in the 12-time periods, has produced an economical benefit $\left(\sum_{t=1}^{T} \Delta \operatorname{Cost}_{t}\right)$ of $\$ 2,386.69$, and the real cost is $\$ 261,311.80$, as shown in the Table IV.

The simulation is then carried our using the proposed strategy by considering the impact of buying and selling to WM given in (2f), where the battery can be working in different capacity during charging and discharging as given in Table I, and the working logic of the batteries is as given in Table II. Table V shows the one-day ( $1^{\text {st }}$ row $)$, one-week $\left(2^{\text {nd }}\right.$ row) and 4-week ( $3^{\text {rd }}$ row) results respectively.

TABLE V Operational Costs Comparison on one day, 7 and 28 days by the 3-bus system as given in the Fig. 6

\begin{tabular}{c|ccccc}
\hline & $\begin{array}{c}\sum_{1}^{T} \text { Cost }_{t} \\
\left(10^{3} \$\right)\end{array}$ & $\begin{array}{c}\sum_{1}^{T} \text { Cost }_{s, t} \\
\left(10^{3} \$\right)\end{array}$ & $\begin{array}{c}\sum_{1}^{T} \text { Prof }_{t}^{\text {sell }} \\
\left(10^{3} \$\right)\end{array}$ & $\begin{array}{c}\sum_{1}^{T} \text { Cost }_{t}^{\text {buy }} \\
\left(10^{3} \$\right)\end{array}$ & $\begin{array}{c}\text { Real Cost } \\
\left(10^{3} \$\right)\end{array}$ \\
\hline 1 & 1749.61 & 1755.21 & 63.23 & 366.15 & $\mathbf{1 7 2 8 . 6 0}$ \\
2 & 10411.10 & 10502.33 & 409.15 & 245.21 & $\mathbf{1 0 3 3 8 . 3 9}$ \\
3 & 43602.00 & 43281.91 & 1024.76 & 1206.13 & $\mathbf{4 3 4 6 3 . 2 8}$ \\
\hline
\end{tabular}

The first column, $\Sigma_{1}^{T} \operatorname{Cost}_{t}$, as given in (1b), represents the reference cost (without batteries and trade) over [1, T]; and, the second column, $\Sigma_{1}^{T} \operatorname{Cost}_{s, t}$, calculated using (4), is the total optimized generation cost of the system equipped with the batteries and trade; the third column is the revenue obtained by selling energy to the market, and the fourth is the expenditure incurred to buy the energy from the market when the price is low. The last column shows the real cost, calculated using (5b), and when this is compared with the reference cost in the first column, it shows that the real cost using the proposed strategy is much less than the reference cost. If more BESS and renewable generators are added, a much less total real cost can be achieved.

\section{B. Revised IEEE 14-bus System}

In this subsection, the computations described in Section $\mathrm{V}-\mathrm{A}$ are repeated for the adapted IEEE 14-bus system as shown in Fig. 7.

Table IV Economical comparison between the 3-bus System with battery and the case without battery over 12-time-slice

\begin{tabular}{|c|c|c|c|c|c|c|c|c|c|c|c|c|c|}
\hline \multirow{2}{*}{$\begin{array}{l}\text { Time } \\
\text { Slice }\end{array}$} & \multicolumn{3}{|c|}{ Data } & \multicolumn{3}{|c|}{ The system without battery } & \multicolumn{5}{|c|}{ The system with battery } & \multicolumn{2}{|c|}{ Statistical results } \\
\hline & $\begin{array}{c}N^{t} \\
\text { MW }\end{array}$ & $\begin{array}{c}D^{t} \\
\text { MW }\end{array}$ & $\begin{array}{c}P^{t} \\
\$ / \mathrm{MWh}\end{array}$ & $\begin{array}{c}\sum_{i=1}^{3} P G_{i}^{t} \\
\text { MW }\end{array}$ & $\begin{array}{l}\operatorname{loss}_{t} \\
\text { MW }\end{array}$ & $\begin{array}{c}\text { Cost }_{t} \\
\$\end{array}$ & $\begin{array}{c}\sum_{i=1}^{3} P G_{s, i}^{t} \\
\quad \text { MW }\end{array}$ & $\begin{array}{l}\operatorname{loss}_{s, t} \\
\text { MW }\end{array}$ & $\begin{array}{l}\Delta R_{j}^{t} \\
\mathrm{MW}\end{array}$ & $\begin{array}{c}R_{j}^{t} \\
\text { MW }\end{array}$ & $\begin{array}{c}\operatorname{Cost}_{s, t} \\
\$\end{array}$ & $\begin{array}{c}\Delta \operatorname{Cost}_{t} \\
\$\end{array}$ & $\sum_{1}^{T} \operatorname{Cost}_{t}(\$)$ \\
\hline 1 & 88 & 378 & 119.98 & 293.40 & 3.36 & 25680.09 & 334.21 & 4.17 & 40 & 80 & 28879.12 & -3199.02 & 26260940 \\
\hline 3 & 84 & 324 & 109.76 & 242.78 & 2.43 & 21883.54 & 303.84 & 3.49 & 60 & 180 & 26462.75 & -4579.20 & \\
\hline 4 & 114 & 308 & 107.84 & 196.22 & 2.19 & 18391.23 & 206.36 & 2.33 & 10 & 190 & 19151.71 & -760.49 & , t \\
\hline 5 & 140 & 300 & 110.10 & 181.72 & 2.44 & 17278.76 & 183.14 & 2.75 & 10 & 200 & 17396.25 & -117.49 & 26121180 \\
\hline 8 & 137 & 336 & 116.32 & 201.43 & 2.62 & 18782.14 & 201.43 & 2.62 & 0 & 200 & 18782.14 & 0 & 0 \\
\hline 9 & 154 & 365 & 131.71 & 213.88 & 3.10 & 19716.00 & 213.88 & 3.10 & 0 & 200 & 19716.00 & 0 & $\sum_{1}^{T} \operatorname{Cost}_{t}^{\text {buy }}$ \\
\hline 10 & 166 & 409 & 152.87 & 246.78 & 3.88 & 22183.43 & 206.09 & 3.19 & -40 & 160 & 19331.91 & 2851.53 & 0 \\
\hline 11 & 164 & 460 & 253.43 & 301.26 & 4.91 & 26269.87 & 219.72 & 3.37 & -80 & 80 & 20874.35 & 5395.52 & Real Cost \$ \\
\hline 12 & 155 & 495 & 150.34 & 345.91 & 5.75 & 32598.77 & 304.97 & 4.81 & -40 & 40 & 26747.77 & 5851.00 & $261,311.80$ \\
\hline
\end{tabular}


In this system, three load buses, bus 11, bus 12 and bus 13, are equipped with the batteries of the same type as given in Sub-section V.A. The cost parameters of all the generators are tripled from the standard version in the 14-bus system to estimate all the practical costs of the grid. To determine the reference cost, the total costs of generation from all generators without the batteries are calculated using OPF $\left(\operatorname{Cost}_{t}\right)$.

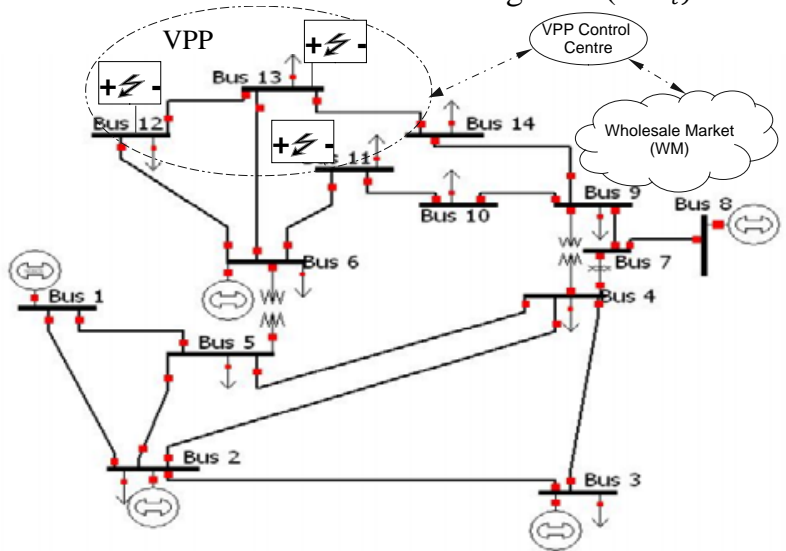

Fig. 7. The IEEE 14 bus system adapted from [22]

In each time slice, whether the battery storage should be charging is decided based on the current price compared to the average price as given in (2b), from which the contribution from each battery can be calculated from (2c) can be obtained. Fig. 8 shows the one-day price data sampled every 30 mins and its average price, and the four levels of the price curve based on the standard deviation. The price stream can be

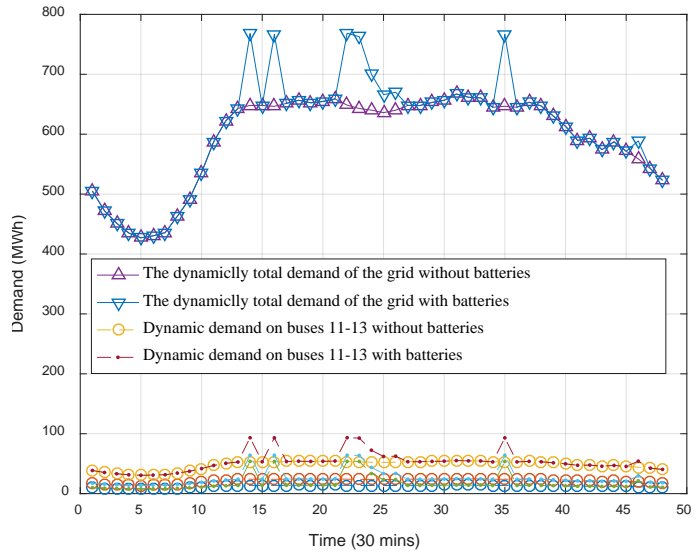

(a)

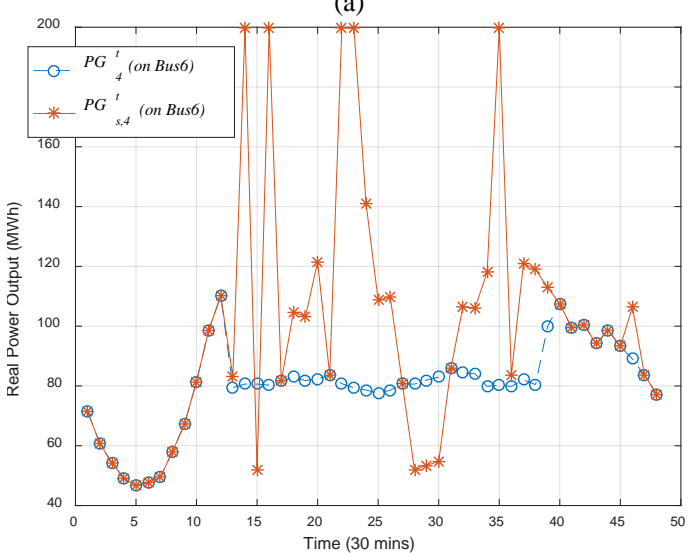

(c) encoded into a consecutive control-signal string consisting of ' $D 2$ ',' $D 1$ ',' $C 1$ ',' $C 2$ ' at the VCC side, and it can be decoded into a serial battery action on the battery side.

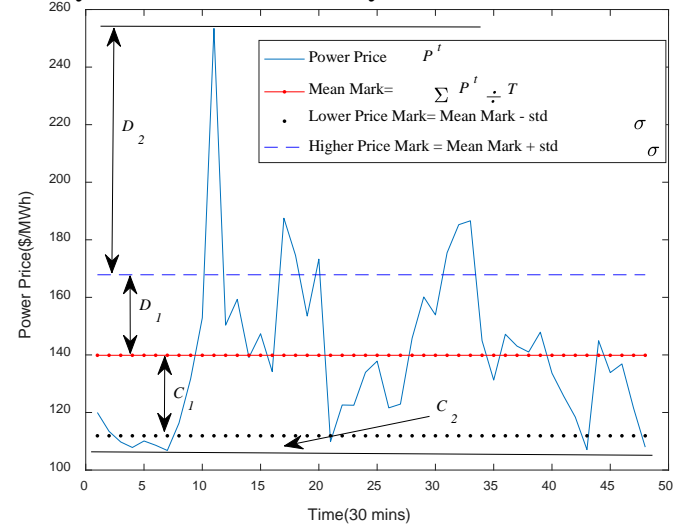

Fig. 8. 24-hour price data sampling per 30 mins

The reference cost $\left(\right.$ Cost $\left._{t}\right)$ in each time slice is used to determine how the battery are used for charging and discharging as shown in Table 1 based on (2e) and (2f).

Fig. 9(a) shows the comparison between the total load demands of buses 11-13 (where the batteries are located) in IEEE 14 bus system with and without the batteries and energy trade. Whenever the batteries are being charged, it becomes an extra load (time slice 14, 16, 22-26, 35, 46) and therefore increases the load demand at that bus. Some of these changes are very small but will be more evident when their costs are calculated. Figs. 9(b) - (d) show the effect of the batteries and

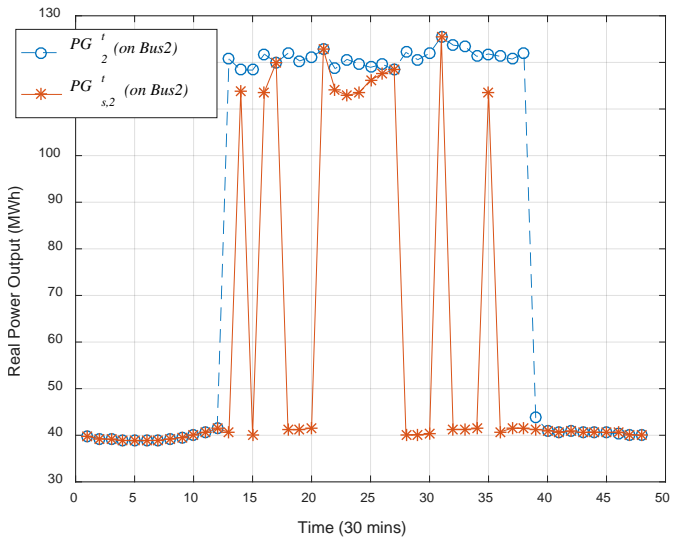

(b)

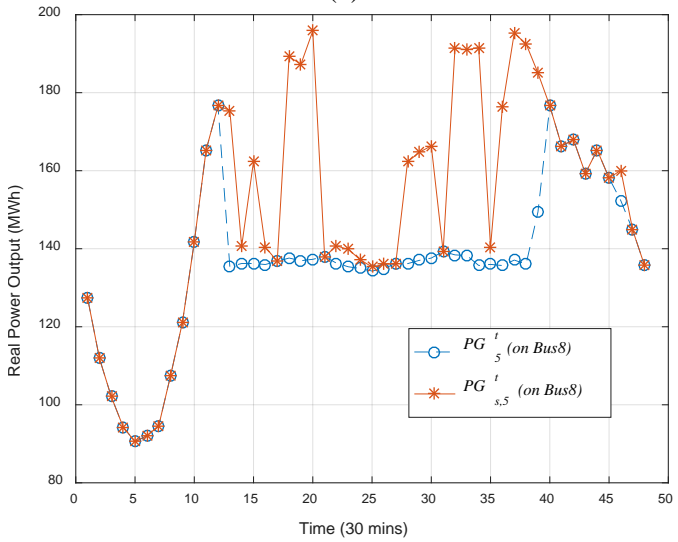

(d)

Fig. 9. Comparison on the dynamic loads and real power outputs in the IEEE revised Case 14 over 1-day data :(a) Dynamic loads on buses 11-13, (b) Dynamic real power output on bus2, (c) Dynamic real power output on bus6, (d) Dynamic real power output on bus8 
the energy trade on the output of the generators of bus 2, bus 6, and bus 8 (bus 1 is the slack node of the system). Fig. 9(b) shows that the generator of bus 2 reduces its output whenever the batteries are being charged (the load demands increase), as shown in Fig. 9(a). Because the cost of generation of this generator is the highest of all the generators, it will be used least to balance the generation and the load. Fig. 9(c) and 9(d) shows that the generation of the other two generators in bus 6 and bus 8 , which are cheaper than the generator of bus 2 . As expected, these two generators carry more impact to balance the generation and the load, as the load is increased due to the charging of the battery as shown in Fig. 9(a). These additional loads also affect the corresponding real power outputs of all other generators. The amounts of these additional loads also depend on whether the batteries are used as energy buyers or temporary loads as shown in Table II

Fig. 10(a) shows the dynamic actions of the battery on bus 11 driven by the spot price shown by the red line based on (2f) and Table I. In the blue bar graph, the parts above the line represent discharging, and the parts below the line represent charging.
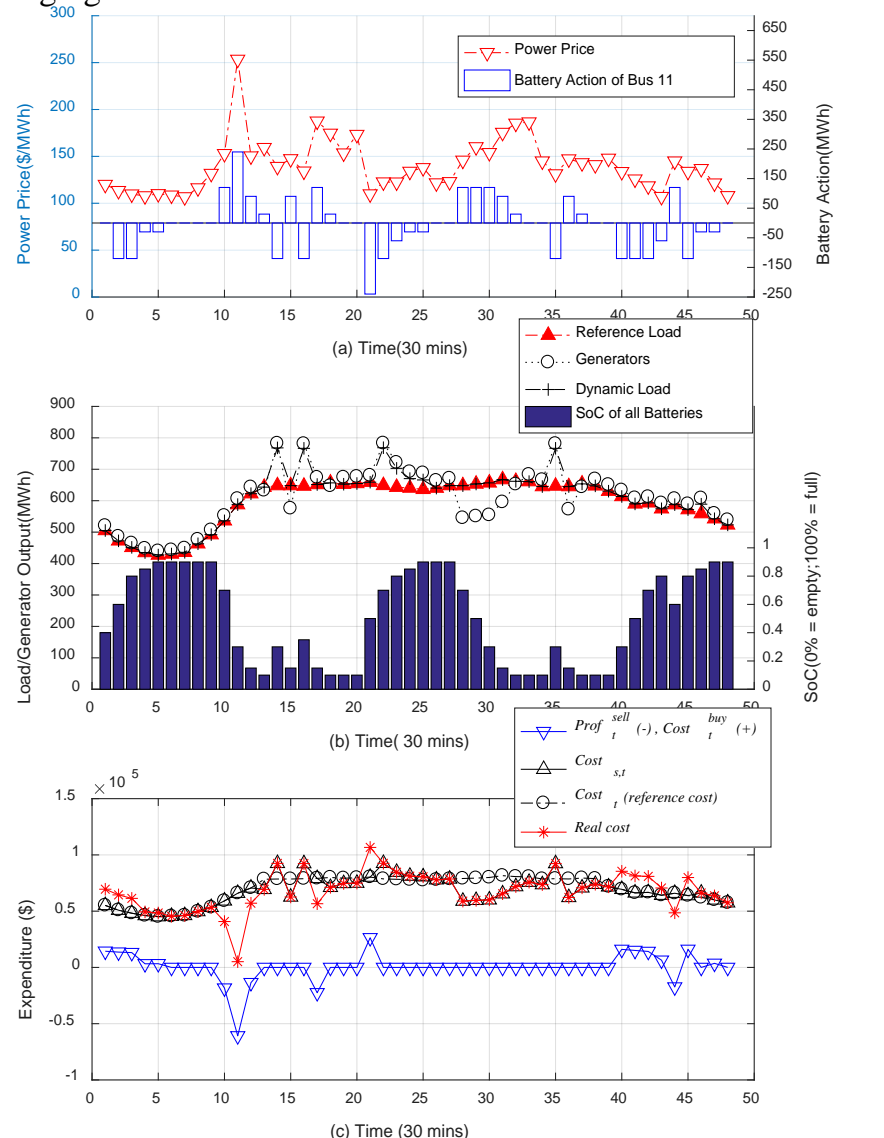

Fig. 10. Dynamic states of the batteries in the Case study 2: (a) Spot price and the battery state on bus 11, (b) Dynamic states including the SoC of the total BESS, the reference load, dynamic real load and the real power output, (c) The dynamic revenue state

In Fig. 10(b), the deep blue bar graph dynamically records the state-of-charge (SoC) of all the batteries in buses 11-13, which increases when the batteries are charged and decreases when the batteries are discharged and are limited with $10 \%$ and $90 \%$. The red triangle line shows the reference load (without batteries and WM).

The purposes of batteries for charging and discharging are defined by (2f) and Table I, e.g., to obtain profit, the batteries are discharged either as energy sellers or as temporary generators; similarly, the batteries can be charged either as energy buyers or as temporary loads. When the batteries are used as energy buyers or energy sellers to WM, the energies are bought or sold to the market and will therefore not affect the local grid, however when the batteries are used as temporary generators or temporary loads, the energies must be supplied or drawn from the local grid. These operations will dynamically affect the load and the generations from other generators.

Fig. 10(b) also shows that depending on whether the batteries are operating as generators when discharging, or they are operating as loads when charging, the load demand and the generations in the local grid are modified as these operations affect the local grid operation. For example, in time slice 15, the batteries are discharging and based on the cost comparison in (2f), the batteries are used as local generators to the local grid, and therefore the total output of all the local generators drops to meet the load demand due to the additional generation from the batteries, similarly in time slice 14 , when the batteries are used as a load to the local grid when charging, the total load demand the total output of the local generator increases, due to the additional load introduced by the batteries.

Fig. 10(b) further shows that depending on whether the batteries are operating as energy sellers when discharging, or they are operating as energy buyers when charging, the load demand and the generations in the local grid are not modified as these operations do not affect the local grid operation. For example, in time slice 10, the batteries are discharging and based on the cost comparison in (2f), the batteries are used as energy sellers to $\mathrm{WM}$, and this operation has no effect on the local grid loads or generations, similarly in time slice 21, when the batteries are used as energy buyers to WM when charging, this operation has no effect on the local grid loads or generations.

Fig. 10(c) shows the reference cost curve, Cost $_{t}$, as a black circle line, the optimized cost for charging and discharging the batteries, $\operatorname{Cost}_{s, t}$, as given in (2) as a black triangle line, the real cost as given in (5b) and the profits and the cost of operating the batteries as energy sellers and energy buyers as given in (2g) and (2h) respectively as a blue triangle line, profit is shown as negative and cost is shown as positive.

For example, in time slice 15, the batteries are discharging, and based on (2f), the decision is to operate the batteries as generators, and hence the total generations from the local generators in the local grid is reduced resulting in a reduced $\operatorname{cost}_{s, t}$, and reduced real cost and since there is no energy trading in the market, there is no profit and loss in trading with the market.

In time slice 10, the batteries are discharging, and based on (2f), the decision is to operate the batteries as energy sellers, and hence $\operatorname{cost}_{s, t}$ is unchanged and the real cost is reduced because of the revenue in selling the energy, and there is there is a profit in selling to WM.

In time slice 14, the batteries are charging, and based on (2f), the decision is to operate the batteries as loads, and hence $\operatorname{cost}_{s, t}$ and real cost are increased and since there is no energy trading in the market, there is no profit and loss in In time slice 21 , the batteries are charging, and based on (2f), the 
decision is to operate the batteries as energy buyers, and hence Cost $_{s, t}$ is unchanged and the real cost is increased because of the cost in buying the energy from WM, and there is there is additional cost is buying from WM.

In time slice 21, the batteries are charging, and based on (2f), the decision is to operate the batteries as energy buyers, and hence Cost $_{s, t}$ is unchanged and the real cost is increased because of the cost in buying the energy from WM, and there is there is additional cost is buying from WM.

In the 48 time slices (half hour interval), the total Cost $_{t}$ is $\$$ $3,340,404.89$, the total Cost $_{s, t}$ is $\$ 3,243,730.50$, the total real cost is $\$ 3,230,765.05$ and the market revenue as defined in Eq. (3) is $\$-12,965.45$. The total revenue as defined in (5a) is $\$$ 83,708.95, which includes the market revenue and the difference between Cost $_{s, t}$ and Cost $_{t}$.

The stimulation is further processed on 1-week and 4week data and the results are shown $1^{\text {st }}$ and $2^{\text {nd }}$ rows respectively in the Table VI. The real costs are much lower than the reference cost.

Table VI Economical comparison on 7 and 28 days using the proposed method for IEEE 14 bus system

\begin{tabular}{ccccc}
\hline $\begin{array}{c}\sum_{1}^{T} \text { Cost }_{t} \\
(\$)\end{array}$ & $\begin{array}{c}\sum_{1}^{T} \text { Cost }_{s, t} \\
(\$)\end{array}$ & $\begin{array}{c}\sum_{1}^{T} \text { Prof }_{t}^{\text {sell }} \\
(\$)\end{array}$ & $\begin{array}{c}\sum_{1}^{T} \text { Cost }_{t}^{\text {buy }} \\
(\$)\end{array}$ & $\begin{array}{c}\text { Real Cost } \\
(\$)\end{array}$ \\
\hline $20,553,251$ & $20,698,442$ & $1,212,662$ & 858,474 & $\mathbf{2 0 , 3 4 4 , 2 5 4}$ \\
$83,113,853$ & $81,805,897$ & $5,554,420$ & $4,059,924$ & $\mathbf{8 0 , 3 1 1 , 4 0 1}$ \\
\hline
\end{tabular}

\section{CONCLUSION}

For solving the multi-period OPF problem modelled on the BESSs of VPP and the market impact, this paper proposes a three-layer deterministic lookahead approach by formalizing the relationship between the BESS actions (such as charging and discharging) and the forecasted price horizon into several finite states and finite price intervals respectively. For this reason, the large possible states in the stochastic optimization (i.e., the multi-period OPF) has reduced effectively. Then a recursive grammar and a non-deterministic finite automaton (NFA) are designed to implement the price-driven strategy. According to the location of the current spot price on the forecasted price horizon, each real-time spot price may transfer the current battery state into another state. Thus, the strict feasibility of the optimal trajectory among the finite states can be ensured. Accordingly, on the VCC side, the continuous spot price can be encoded into a consecutive control symbol string, which can be received and decoded in time on the battery side to operate the battery as a local generator, a local load, an energy buyer, an energy seller, or in an idle state. The proposed method has two main tasks: one is to minimize the operational cost, and the other is to maximize the profit when the VPP is operating in the electricity market. Simulation results have revealed that this prototype is promising for real-time control and it is cost-effective for implementation. Further works are required to enhance the energy efficiency, operation flexibility and economic revenue in the grid-connected environment.

\section{REFERENCES}

[1] M. Ross, C. Abbey, F. Bouffard, and G. Joos, "Microgrid economic dispatch with energy storage systems," IEEE Transactions on Smart Grid, vol. 9, no. 4, pp. 3039-3047, 2018.

[2] K. Wang, X. Hu, H. Li, P. Li, D. Zeng, and S. Guo, "A Survey on Energy Internet Communications for Sustainability," IEEE Transactions on Sustainable Computing, 2017, vol.2,no.3,pp.231-254.

[3] J. Carpentier, "Contribution to the economic dispatch problem," (in French) Bulletin de la Societe Francoise des Electriciens, vol. 3, no. 8, pp. 431-447, 1962.

[4] W. B. Powell and S. Meisel, "Tutorial on stochastic optimization in energy_Part I: Modeling and policies," IEEE Transactions on Power Systems, vol. 31, no. 2, pp. 1459-1467, 2016.

[5] W. B. Powell and S. Meisel, "Tutorial on stochastic optimization in energy-Part II: An energy storage illustration,” IEEE Transactions on Power Systems, vol. 31, no. 2, pp. 1468-1475, 2016.

[6] F. Hafiz, D. Lubkeman, I. Husain, and P. Fajri, "Energy storage management strategy based on dynamic programming and optimal sizing of PV panel-storage capacity for a residential system,” in 2018 IEEE/PES Transmission and Distribution Conference and Exposition (T\&D), 2018, pp. 1-9.

[7] R. Halvgaard, L. Vandenberghe, N. K. Poulsen, H. Madsen, and J. B. Jørgensen, "Distributed model predictive control for smart energy systems," IEEE Transactions on Smart Grid, vol. 7, no. 3, pp. 16751682, 2016.

[8] D. Grune, K. Van Reeuwijk, H. E. Bal, C. J. Jacobs, and K. Langendoen, Modern compiler design. Springer Science \& Business Media, 2012.

[9] A. V. Aho, R. Sethi, and J. D. Ullman, "Compilers, principles, techniques,” Addison wesley, vol. 7, no. 8, p. 9, 1986.

[10] G. Pola and M. D. Di Benedetto, "Symbolic models and control of discrete-time piecewise affine systems: An approximate simulation approach," IEEE Transactions on Automatic Control, vol. 59, no. 1, pp. 175-180, 2014

[11] Y. Guo, K. Baker, E. Dall'Anese, Z. Hu, and T. H. Summers, "Databased distributionally robust stochastic optimal power flow-Part I: Methodologies,” IEEE Transactions on Power Systems, vol. 34, no. 2, pp. 1483-1492, 2018.

[12] Y. Guo, K. Baker, E. Dall'Anese, Z. Hu, and T. H. Summers, "DataBased Distributionally Robust Stochastic Optimal Power Flow-Part II: Case Studies,” IEEE Transactions on Power Systems, vol. 34, no. 2, pp. 1493-1503, 2018.

[13] D. Gayme and U. Topcu, "Optimal power flow with large-scale storage integration,” IEEE Transactions on Power Systems, vol. 28, no. 2, pp. 709-717, 2013

[14] S. Singh, M. Singh, and S. C. Kaushik, "Optimal power scheduling of renewable energy systems in microgrids using distributed energy storage system,” IET Renewable Power Generation, vol. 10, no. 9, pp. 13281339, 2016.

[15] M. Ross, C. Abbey, F. Bouffard, and G. Joos, "Microgrid economic dispatch with energy storage systems," IEEE Transactions on Smart Grid, vol. 9, no. 4, pp. 3039-3047, 2016.

[16] C. Xiao, Z. Dong, Y. Xu, K. Meng, X. Zhou, and X. Zhang, "Rational and self-adaptive evolutionary extreme learning machine for electricity price forecast,” Memetic Computing, vol. 8, no. 3, pp. 223-233, 2016.

[17] https://www.aemo.com.au/Electricity/

[18] X. Chen, B. Jones, M. Becchi, and T. Wolf, "Picking pesky parameters: Optimizing regular expression matching in practice," IEEE Transactions on Parallel and Distributed Systems, vol. 27, no. 5, pp. 1430-1442, 2016.

[19] A. Ordono, E. Unamuno, J. A. Barrena, and J. Paniagua, "Interlinking converters and their contribution to primary regulation: a review," International Journal of Electrical Power \& Energy Systems, vol. 111, pp. 44-57, 2019.

[20] J. Liu, N. Zhang, C. Kang, D. Kirschen, and Q. Xia, "Cloud energy storage for residential and small commercial consumers: A business case study," Applied energy, vol. 188, pp. 226-236, 2017.

[21] R. D. Zimmerman and C. E. Murillo-Sanchez, "MATPOWER Optimal Scheduling Tool MOST 1.0 User's Manual”, 2016. [Online]. Available: http://www.pserc.cornell.edu/matpower MOST-manual.pdf.

[22] ICSEG, "Illinois center for a smarter electric grid (icseg)." [Online]. Available: http://icseg.iti.illinois.edu/ieee-14-bus-system/. 